\title{
Más aristas que solo el fraude: el caso de Dololed en Colombia
}

\section{More edges than just fraud: Dololed's case in Colombia}

\author{
Israel Rico-Alba ${ }^{1}$ \\ Forma de citar: Rico-Alba I. Más artistas que solo el fraude: el caso de Dololed en Colombia. Salud UIS. 52(4): $492-493$. \\ doi: https://doi.org/10.18273/revsal.v52n4-2020018 (c) (i)
}

Carta al editor de la revista Salud UIS sobre el artículo de Stashenko, et al. Titulado "Hallazgo de diclofenaco en un producto fitoterapéutico a base de caléndula comercializado en Colombia".

Se plantean las consecuencias, retos y oportunidades del sistema de vigilancia de fitofármacos falsificados.

Los productos médicos falsificados y de calidad subestándar son un riesgo para la salud global. En consecuencia, las autoridades sanitarias nacionales implementan mecanismos de vigilancia para detectar medicamentos, vacunas y productos de diagnóstico con éstas características ${ }^{1}$. Sin embargo, el uso de estos mecanismos en productos como los fitofármacos es menos frecuente. La amplitud de resultados analíticos presentados en el caso de Dololed ${ }^{\mathrm{O} 2}$; así como la forma en la que se identificaron las señales de alarma del producto y el manejo por parte del Instituto Nacional de Vigilancia de Medicamentos y Alimentos (INVIMA) deja lecciones globales para mejorar el monitoreo y uso seguro de otros fitofármacos.

El estudio de Stashenko, et al. identificó 3 grupos de productos en las tabletas analizadas: terapéuticos (diclofenaco), fitofármacos no terapéuticos o terapéuticos a dosis insuficientes para alcanzar un efecto armacológico e impurezas. El que un fitofármaco "natural" contenga un producto de síntesis química como diclofenaco representa un fraude al consumidor. Sin embargo, también representa un beneficio importante, así como retos y riesgos. La dosis promedio de diclofenaco cuantificada en el estudio permite que sus consumidores alcancen un efecto analgésico. Justo el beneficio esperado por los consumidores al adquirir la caléndula. Datos del fabricante reportaron una venta mensual de 100000 unidades $^{3}$. Esto refleja, la necesidad de analgesia con productos no farmacológicos que satisfacía Dololed $^{\circ}$ en la población. Ahora que se retiró el producto del mercado los consumidores podrían buscar otros fitofármacos como substitutos terapéuticos. Un reto para el INVIMA será garantizar la seguridad y efectividad de estos substitutos para no dejar a la población con una necesidad insatisfecha. Con la combinación de diclofenaco más caléndula apareció un reto adicional. Actualmente, no existen estudios que demuestren la eficacia analgésica de la caléndula ${ }^{1,4}$; por tanto, al combinarse con un verdadero analgésico se mantendría la percepción de que el fitofármaco funciona. Ello inhibe o retrasa la detección de la señal de "falta de eficacia" del producto por parte de los mecanismos de vigilancia tradicional. Incluso, ésta podría ser una estrategia deliberada de quién elabora productos fraudulentos para dificultar su identificación; la cual podría ocurrir en casos similares. Finalmente, la presencia de diclofenaco, así como otros fitofármacos e impurezas, son un riesgo. Sin información precisa y completa sobre el contenido de cada tableta las personas no pueden advertir y evaluar correctamente el riesgo al que se exponen.

La atención puesta en este producto surgió del reporte de eventos adversos provistos por un número pequeño de consumidores. Sin duda, éste un recordatorio de la importancia de crear un ambiente de responsabilidad compartida para la vigilancia de productos médicos en donde todos participen: prescriptores, autoridades sanitarias, consumidores, fabricantes y comercializadores del insumo. Más aún en el tema de fitofármacos analgésicos en donde existen una calidad subestándar en muchos productos y la evidencia del beneficio es baja ${ }^{4-6}$. Esto invita a pensar que no hay señales pequeñas.

1. Universidad Nacional Autónoma de México, CDMX, México.

Correspondencia: Israel Rico-Alba. Dirección:Dragon 121, Col. Prado Churubusco, Coyoacán. C.P. 04230, CDMX, México. Teléfono: +52 5540341887. Correo electrónico: mdricoalba@yahoo.com.mx 
Los mecanismos de vigilancia nacionales establecen que las señales, eventos adversos y prácticas de manufactura de los laboratorios deben ser evaluadas 7 . Interesantemente, un producto con 7 años en el mercado tuvo solo cuatro evaluaciones: la primera hasta los 4 años de su aprobación y las 3 siguientes de forma anual. Si bien los análisis del INVIMA previos al 2020 no detectaron diclofenaco, si identificaron "productos diferentes a los autorizados" en 2017 y "diferencias sustanciales en los resultados entre muestras analizadas del laboratorio fabricante" en $2019^{8}$. Estos resultados muestran problemas en la calidad del producto por parte del productor quien tiene la responsabilidad de estandarizar sus procesos para seguir operando. En enero del 2020 el INVIMA identificó diclofenaco en muestras analizadas del productor y puntos de venta por lo que el fitofármaco fue retirado del mercado. Esto muestra que el proceso de monitoreo actual es insuficiente para detectar y controlar los riesgos a tiempo. Se requiere un seguimiento más frecuente y estandarizado, menos laxo. Y también herramientas más potentes. El extenso análisis presentado por Stashenko, et al. ejemplifica que obtener estos resultados requiere capacidades técnicas y recursos que no están al alcance de todos. De ahí que la colaboración de los distintos participantes es toral.

Los fitofármacos son percibidos como productos eficaces con pocos o ningún evento adverso 9 . Casos como el de Dololed $^{\odot}$ afectan la confianza que se tienen en alternativas terapéuticas y culturales importantes en países como Colombia. Así mismo, dañan la confianza en sus productores, prescriptores y entidades sanitarias que los regulan y proveen. El que un producto contenga un medicamento no declarado o una impureza es tan grave como presentar una calidad heterogénea. Los diferentes participantes en el caso tuvieron un denominador común: todos actuaron tarde. Para recobrar la confianza de los consumidores se debe actualizar el marco de acción regulatorio de todos los involucrados. Apoyarse en los avances que ya existen en otras partes del mundo, incluyendo incorporar mejores herramientas analíticas a los mecanismos de vigilancia, pueden ser de utilidad como un primer paso ${ }^{10}$. Si el problema de la falsificación y calidad subóptima de productos médicos es un problema de salud global, entonces, se requieren de respuestas homogéneas y globales para enfrentarlo.

\section{Referencias}

1. OMS. Productos médicos de calidad subestándar y falsificados. Online: https://www.who.int/es/news-room/ fact-sheets/detail/substandard-and-falsified-medical-products

2. Statshenko E, González A, Martínez J, Durán C, Oliveros C, Gualteros LK, et al. Hallazgo de diclofenaco en un producto fitoterapéutico a base de caléndula comercializado en Colombia. Salud UIS; 2020; 52(3): 261-284. doi: http://dx.doi.org/10.18273/revsal.v52n3-2020008

3. Unidad investigativa. Dololed no va más, su dueño decidió frenar su producción. El tiempo. https://www. eltiempo.com/unidad-investigativa/dueno-de-dololed-decidio-frenar-su-produccion-457314

4. Di Lorenzo C, Dell'Agli M, Badea M, Dima L, Colombo E, Sangiovanni E, et al. Plan food supplements with anti-inflammatory properties: a systematic review (II). Crit Rev Food Sci Nutr. 2013; 53(5): 507-516. doi: $10.1080 / 10408398.2012 .691916$

5. Del Grossi-Moura M, Cruz-Lopes L, Biavatti MW, Kennedy SA, de Oliveira e Silva MC, Silva MT, et al. Oral herbal medicines marketed in Brazil for the treatment of ostheoarthritis: A systematic review and meta-analysis. Phytother Res. 2017; 31(11): 1676-1685. doi: 10.1002/ptr.5910

6. Pan S, Neeraj A, Srivastava KS, Kishore P, Danquah MK, Sarethy IP. A Proposal for a Quality System for Herbal Products. J Pharm Sci. 2013; 102(12): 4230-4241. doi: 10.1002/jps.23732

7. Invima. Resolución 2266 Por el cual se reglamentan los regímenes de registros sanitarios, y de vigilancia y control sanitario y publicidad de los productos fitoterapéuticos. https://www.invima.gov.co/documents/20143/453792/ decreto_2266_2004.pdf

8. Invima. Aclaraciones frente a la eventual presencia de diclofenaco en el producto fitoterapéutico caléndila officinalis marca DOLOLED. https://www.invima.gov.co/aclaraciones-frente-a-la-eventual-presencia-dediclofenaco-en-el-producto-fitoterapeutico-calendula-officinalis-marca-dololed

9. OMS. Medicina tradicional: definiciones. https://www.who.int/topics/traditional_medicine/definitions/es/

10. Qu L, Zou W, Wang Y, Wang M. European regulation model for herbal medicine: The assessment of the EU monograph and the safety and efficacy evaluation in marketing authorization or registration in Member States. Phytomedicine 2018; 42, 219-225. doi: 10.1016/j.phymed.2018.03.048 